\title{
Pratiques
}

Linguistique, littérature, didactique

187-188 | 2020

Enseignement du texte littéraire dans l'espace

francophone: pratiques, formation, recherche

\section{Quel travail sur les textes littéraires dans les écoles québécoises de l'entrée à la sortie du secondaire?}

Olivier Dezutter, Julie Babin et Martin Lépine

\author{
(2) OpenEdition \\ Journals \\ Édition électronique \\ URL : https://journals.openedition.org/pratiques/8962 \\ DOI : $10.4000 /$ pratiques.8962 \\ ISSN : 2425-2042 \\ Éditeur \\ Centre de recherche sur les médiations (CREM)
}

Référence électronique

Olivier Dezutter, Julie Babin et Martin Lépine, «Quel travail sur les textes littéraires dans les écoles québécoises de l'entrée à la sortie du secondaire? », Pratiques [En ligne], 187-188|2020, mis en ligne le 12 décembre 2020, consulté le 20 juillet 2021. URL : http://journals.openedition.org/pratiques/8962 ; DOI : https://doi.org/10.4000/pratiques.8962

Ce document a été généré automatiquement le 20 juillet 2021.

(c) Tous droits réservés 


\title{
Quel travail sur les textes littéraires dans les écoles québécoises de l'entrée à la sortie du secondaire?
}

\author{
Olivier Dezutter, Julie Babin et Martin Lépine
}

\section{Contexte}

1 La lecture des textes littéraires est une activité inscrite au sein des pratiques régulières dans les classes de français aux différentes étapes de la scolarité. Elle est conditionnée par un certain nombre d'éléments, dont les conditions matérielles de travail et les orientations des programmes officiels qui déterminent les apprentissages à réaliser aux différentes étapes de la scolarité. Au Québec, les enseignant·es disposent toutefois d'une marge de manœuvre assez large pour ce qui concerne le choix des corpus, les modes d'approche des textes et les activités à privilégier. Contrairement à ce qui prévaut dans d'autres pays, le concept d'approche analytique est absent des programmes officiels.

2 Dans cette contribution, nous proposons un regard sur la manière dont les programmes de français actuellement en vigueur pour l'enseignement au secondaire, en particulier, mais aussi pour les ordres d'enseignement qui précèdent (l'école primaire) et qui suivent (l'enseignement collégial), encadrent la question de la lecture des textes littéraires et du travail effectué autour de ces textes, en essayant de dégager certains indices de progression. Pour faire écho à ces éléments du curriculum prescrit, nous levons également un coin du voile sur une partie du curriculum enseigné, en prenant appui sur un ensemble de données empiriques recueillies dans le cadre de nos recherches étalées sur les quinze dernières années. Mais avant tout, en guise de prémisses, nous partageons notre vision de ce qui fait la spécificité du travail sur les textes littéraires dans le contexte scolaire. 


\section{Les caractéristiques du travail sur les textes littéraires à l'école}

3 La constitution de la lecture des textes littéraires en " tâche scolaire » se concrétise par un certain nombre de choix qui concernent à la fois la finalité de ce volet constitutif de l'enseignement-apprentissage de la langue, les formats et contenus des textes donnés à lire, les activités qui accompagnent la lecture de ces textes ainsi que les éléments à évaluer pour rendre compte des apprentissages effectués par les élèves.

4 À travers sa longue histoire, l'école a fabriqué un certain nombre d'« objets » de lecture particuliers qui, en quelque sorte, "formatent» le rapport des élèves aux textes littéraires et à la lecture littéraire. Les textes donnés à lire dans le cadre des cours de français sont présentés sous une diversité de formats matériels : des extraits isolés, des extraits rassemblés dans un manuel ou dans une anthologie, des textes brefs (conte, nouvelle, chanson, poème, sketch...) extraits d'un recueil, ou encore des œuvres complètes ${ }^{1}$, de longueur variable, éditées avec ou sans appareillage didactique.

5 Dans l'histoire de la discipline "français ", chaque format de texte est associé à des modes de lecture, des approches et des dispositifs didactiques spécifiques qui vont de pair avec certains types d'activités, tant pour l'enseignante que pour les élèves. Ainsi, par exemple, le commentaire de texte est un exercice scolaire centré sur l'extrait alors que le journal de lecture, la fiche de lecture, le traditionnel résumé écrit de livre (comme l'indique son appellation), ou encore l'exposé oral sont le plus souvent associés à la lecture d'une œuvre complète. En effet, ces derniers nécessitent la combinaison d'une présentation d'informations relatives à l'auteur et au contexte de création de l'œuvre, d'un résumé de l'intrigue et d'une éventuelle appréciation personnelle, comme l'ont montré des enquêtes réalisées en contexte québécois (Dezutter et al., 2012).

6 À l'instar de l'ensemble des autres pratiques d'enseignement, le travail sur les textes littéraires est, en partie, conditionné par le cadre fixé par les instructions officielles qui fournissent des indications relatives à la sélection des corpus ou aux compétences à développer et à évaluer, aux savoirs à enseigner et à certains types d'activités à mettre en œuvre, comme nous le verrons dans la suite de cet article. Les pratiques sont aussi fortement influencées par les épreuves évaluatives, qui jouent un rôle crucial dans la certification des élèves (Popham, 2001) et qui se sont démultipliées au cours des dernières années, à la demande de diverses instances (ministère de l'Éducation, commissions scolaires). Au Québec, l'analyse des textes littéraires est au cœur de l'épreuve unique de français dont la réussite est indispensable pour l'obtention du diplôme d'études collégiales² (Ministère de l'Éducation, de l'Enseignement supérieur et de la Recherche, 2015). De ce fait, à cet ordre d'enseignement, trois cours de «français, langue et littérature " sur quatre s'orientent vers une rédaction de type commentaire composé ou dissertation, en vue de l'épreuve de sanction menant à la diplomation. L'épreuve finale du secondaire est de nature bien différente et sans relation avec la lecture de textes littéraires puisqu'il s'agit de produire un texte argumentatif en lien avec un sujet d'actualité, sur la base d'un dossier comprenant des textes courants ${ }^{3}$.

7 Tant pour le choix des œuvres que pour le choix des manuels ou l'élaboration de certaines épreuves évaluatives en lecture non centralisées au niveau provincial, les enseignante's de la plupart des écoles secondaires sont invitées à se concerter entre collègues du même niveau ou du même cycle ${ }^{4}$. Cette concertation, en particulier pour ce qui concerne la préparation des épreuves communes d'évaluation en lecture, tend à uniformiser les approches analytiques des textes, en privilégiant un questionnement 
axé sur les composantes de la compétence de lecture spécifiées dans les instructions officielles, que nous présentons plus loin $^{5}$. Ceci aboutit souvent à structurer le questionnaire d'examen en quatre parties distinctes portant sur la compréhension, l'interprétation, la réaction et l'appréciation, comme le prescrit le cadre sur l'évaluation du ministère (Ministère de l'Éducation, du Loisir et du Sport, 2011b).

8 Selon S. Florey, N. Cordonier, C. Ronveaux et S. E. Harmassi (2015), le travail sur les textes littéraires en contexte scolaire se situe à l'intersection de l'apprentissage de la lecture et de l'apprentissage de la lecture de la littérature, auxquels on pourrait ajouter l'apprentissage de l'écriture. Ce travail peut être orienté selon diverses finalités que S. Richard (2006) propose de distinguer en quatre ordres:

- cognitivo-langagier ;

- psychoaffectif

- esthético-culturel

- social et politique.

9 Ces finalités ne sont bien sûr pas exclusives, mais chacune d'elle va de pair avec une centration sur certains types de savoirs à acquérir. K. Canvat (2005) fait état à ce sujet, de savoirs socio-institutionnels, formels et historiques. J. Babin (2016), pour sa part, a repéré que, dans les pratiques des enseignante's relatives à la lecture d'œuvres complètes à l'ordre collégial dans le cadre des cours de Français, langue et littérature, la mobilisation de savoirs sur la littérature, sur les processus de lecture, sur le monde et sur la langue sont des savoirs qui recoupent différents « angles d'entrée dans l'œuvre ». Ainsi, selon les pratiques qu'elle a observées dans plusieurs classes, un angle d'entrée esthétique entrainera presque de facto une insistance plus grande lors du travail d'analyse de l'œuvre sur les savoirs sur la langue et la littérature, alors qu'un angle d'entrée historique pourrait solliciter davantage de savoirs sur le monde, par exemple.

10 Après avoir souligné ces quelques éléments qui caractérisent le travail sur les textes littéraires en contexte scolaire, venons-en au cœur de cet article, à savoir le repérage des indices de progression tels qu'ils sont prescrits dans les instructions officielles des différents ordres d'enseignement et tel qu'il est possible de les reconstituer en contexte de classe à partir d'une mise en commun de résultats d'un ensemble de recherches empiriques réalisées dans les différents ordres d'enseignement au Québec au cours des vingts dernières années.

\section{La vision des instructions officielles}

$11 \mathrm{Au}$ Québec, les instructions officielles relatives à l'enseignement du français au primaire et au secondaire en vigueur encore actuellement datent de la mise en place de ce qui a été appelé la "réforme de l'éducation » au tournant des années 2000. Cette réforme se caractérisait par l'introduction de l'approche par compétences dans toutes les disciplines scolaires ${ }^{6}$ et par la revalorisation de la dimension culturelle de toutes les disciplines enseignées (Ministère de l'Éducation, du Loisir et du Sport, 2001, 2006, 2009b). Pour la discipline "français langue d'enseignement ", cela s'est traduit entre autres par des exigences plus fortes pour ce qui concerne le nombre d'œuvres obligatoires à lire au secondaire (Dezutter et al., 2012).

12 Les programmes pour le primaire et le secondaire présentent une vision un peu différente de la compétence en lecture. Cette compétence est scindée en deux dans le programme du primaire, lequel distingue : d'une part, la compétence «Lire des textes 
variés ", qui inclut la lecture de textes courants et littéraires; et d'autre part, la compétence "Apprécier des œuvres littéraires", présentée comme le lieu d'orchestration et de synthèse des compétences à lire, à écrire et à communiquer oralement, en français ${ }^{7}$. Ces deux compétences sont fondues en une seule compétence dans le programme pour le secondaire : «Lire et apprécier des textes variés ».

Les programmes du Québec ont pour point commun une grande marge de manœuvre laissée aux enseignant·e's à propos de la sélection des corpus et du choix des activités liées à la lecture des textes. Selon le programme du primaire, les élèves devraient être mis « quotidiennement en contact avec des œuvres nombreuses et variées » (Ministère de l'Éducation, du Loisir et du Sport, 2001, p. 84), c'est-à-dire de différents genres et sur divers supports. Au secondaire, les enseignant-e's doivent prévoir un programme de lecture de dix œuvres littéraires complètes par année, dont 5 œuvres narratives et 5 œuvres complémentaires, qui peuvent être des poèmes, chansons, pièces de théâtre, monologues, œuvres illustrées ou adaptations cinématographiques. Pour établir ce programme, il s'agit de tenir compte les critères généraux suivants : variété d'auteur.e's et d'univers, équilibre entre les œuvres écrites par des hommes et celles écrites par des femmes, priorité aux œuvres contemporaines avec ouverture sur celles du passé, accent sur les œuvres de la littérature québécoise par rapport à celles de France ou du reste du monde (Ministère de l'Éducation, du Loisir et du Sport, 2006, 2009b).

Plus récemment, un document présentant la Progression des apprentissages (Ministère de l'Éducation, du Loisir et du Sport, 2009c pour le primaire ; Ministère de l'Éducation, du Loisir et du Sport, 2011a pour le secondaire) est venu, entre chaque cycle, baliser davantage la répartition des contenus propres à chacune des compétences à développer, ce qui a entrainé, pour la lecture, une répartition des genres à lire et des contenus spécifiques à aborder selon les différentes années, comme on peut le voir dans le tableau 1 ci-dessous, présentant la répartition des genres narratifs devant être approfondis chaque année au secondaire. Il faut noter que cette division ne repose sur aucun argument théorique explicite: comment justifier le fait d'avoir, par exemple, choisi de réserver la fable à la $2^{\mathrm{e}}$ année du secondaire (fable qui devrait déjà avoir été maitrisée en $5^{\mathrm{e}}$ année du primaire) et le conte à la $3^{\mathrm{e}}$, ou encore le roman policier à la $2^{\mathrm{e}}$ année et le roman historique à la $3^{\mathrm{e}}$ ? On peut inférer que, parmi les critères de décision, ont figuré la longueur du texte, son degré de complexité narrative ou encore certaines thématiques traitées dans certains genres comme le roman sociologique, programmé en dernière année du secondaire. Il faut ajouter que la répartition rapportée dans le tableau 1 est relativement proche de celle qui était prescrite dans un programme d'études précédent (Ministère de l'Éducation du Québec, 1980), à laquelle les enseignant·e·s s'étaient habituée·s. avec les années. 
Tableau 1 : Répartition des genres à travailler au secondaire

\begin{tabular}{|c|c|c|c|}
\hline \multicolumn{4}{|c|}{ Les genres narratifs } \\
\hline & Lecture & Écriture & Communication orale \\
\hline \multirow{2}{*}{$\begin{array}{l}1^{\text {er }} \\
\text { cycle }\end{array}$} & $\begin{array}{l}\text { 1re }^{\text {re }} \text { secondaire } \\
\text { Album, bande dessinée; roman } \\
\text { (psychologique }{ }^{2} \text {, d'aventures ou de } \\
\text { fantaisie }^{3} \text { ) }\end{array}$ & $\begin{array}{l}\text { 1re secondaire } \\
\text { \# Pastiche d'une description } 4 \text { de lieu } \\
\text { dans un récit (voir Description) } \\
\text { " Épisode d'un récit (d'aventures ou } \\
\text { de fantaisie) inspiré d'un livre ou } \\
\text { d'un film }\end{array}$ & $\begin{array}{l}1^{\text {re }} \text { secondaire } \\
\text { E Écoute d'un récit }\end{array}$ \\
\hline & $\begin{array}{l}2^{\text {e }} \text { secondaire } \\
\text { = Fable, récits de genres variés, } \\
\text { roman (policier ou psychologique) }\end{array}$ & $\begin{array}{l}\text { 2e secondaire } \\
\text { - Récit policier ou psychologique } \\
\text { dans lequel sont caractérisés des } \\
\text { personnages et leurs actions ou } \\
\text { adaptation d'une fable }\end{array}$ & $\begin{array}{l}\text { 2e secondaire }^{\text {en Écoute d'une fable }} \\
\text { - E }\end{array}$ \\
\hline \multirow{3}{*}{$\begin{array}{l}2^{\mathrm{e}} \\
\text { cycle }\end{array}$} & $\begin{array}{l}\text { 3e secondaire } \\
\text { = Conte (fantastique, moderne ou } \\
\text { traditionnel), légende, mythe ou } \\
\text { récit de création } 5 \\
\text { = Roman historique ou roman de } \\
\text { chevalerie }\end{array}$ & $\begin{array}{l}\text { 3e secondaire } \\
\text { - Conte original ou transformé } \\
\text { (fantastique, moderne ou } \\
\text { traditionnel) ou récit de création }\end{array}$ & $\begin{array}{l}\text { 3e secondaire } \\
\text { - Écoute d'un conte } \\
\text { ou d'une légende }\end{array}$ \\
\hline & $\begin{array}{l}\text { 4e secondaire } \\
\text { a Nouvelle ou roman (fantastique, } \\
\text { initiatique, psychologique, de } \\
\text { science-fiction) }\end{array}$ & $\begin{array}{l}\text { 4e }^{\text {e secondaire }} \\
\text { = Nouvelle ou récit (fantastique, } \\
\text { psychologique ou de science-fiction) } \\
\text { original ou inspiré d'un film }\end{array}$ & $4^{e}$ secondaire \\
\hline & $\begin{array}{l}5^{\text {e }} \text { se condaire } \\
\text { = } \text { CEuvres littéraires engagées : } \\
\text { roman, bande dessinée, chanson, } \\
\text { fable, monologue, pièce de théâtre, } \\
\text { poésie } \\
\text { - Roman psychologique ou } \\
\text { sociologique }\end{array}$ & $\begin{array}{l}5^{e} \text { secondaire } \\
\quad \text { - Récit avec contrainte }{ }^{6}\end{array}$ & $\begin{array}{l}\text { 5e }^{\mathrm{e}} \text { secondaire } \\
\text { = Écoute d'une } \\
\text { œuvre littéraire } \\
\text { adaptée à l'écran }\end{array}$ \\
\hline
\end{tabular}

Source : Ministère de l'Éducation, du Loisir et du Sport (2011a, p. 28).

15 En examinant la Progression des apprentissages de chacun des ordres d'enseignement, on peut repérer dans les contenus liés à la narration, présentée comme traversant les compétences lire-écrire et communiquer oralement, un certain amalgame entre différentes approches des textes, qui ne sont pas explicitement mises en relation les unes avec les autres :

- l'approche communicative, qui conduit à repérer les éléments constitutifs de "la situation de communication " (identifier l'auteur du texte, se situer en tant que lecteur ou auditeur de textes littéraires) (Ministère de l'Éducation, du Loisir et du Sport, 2009b, p. 29) ;

- l'approche narratologique, avec encore une présence marquée de l'analyse narratologique du roman (Genette, 1972 ; Greimas, 1966) : distinguer l'auteur du narrateur (au secondaire), constater la succession (séquence) des évènements dans l'intrigue (au primaire) ; éléments du schéma actantiel (adjuvant, opposant...) ;

- l'approche linguistique: attention portée au type de vocabulaire utilisé («vocabulaire neutre ou expressif pour désigner ou caractériser la voix, les tics, les traits de caractère, les sentiments ; le statut social », (Ministère de l'Éducation, du Loisir et du Sport, 2011a, p. 30); repérage de figures de style (comparaison, énumération ( $1^{\text {re }}$ secondaire), métaphore $\left(2^{\mathrm{e}} \mathrm{sec}.\right)$, gradation, répétition $\left(3^{\mathrm{e}} \mathrm{sec}\right.$.) ;

- l'approche thématique : dégager les valeurs présentées (ex : entraide, générosité, altruisme au primaire) ou la vision du monde (secondaire);

- l'approche socioculturelle et historique : prendre en compte le contexte de production et de réception d'un texte ;

- l'approche sociologique de l'institution littéraire: connaissances relatives au fonctionnement de l'institution littéraire et au «monde du livre : processus d'édition et de diffusion ». 
16 À ces approches s'ajoute ou se superpose en quelque sorte l'approche générique. S'inscrivant dans des "modes de discours", les genres ayant été choisis "comme organisateur[s] des compétences langagières » (Chartrand, Émery-Bruneau \& Sénéchal, 2015 [2013]) En effet,

[c]es derniers structurent le message d'une façon particulière selon le genre de texte et ils privilégient certaines ressources langagières. L'essentiel du contenu de chaque mode de discours est donc constitué d'un tableau des genres et d'un tableau des contenus d'apprentissage dans lequel on retrouve les stratégies, notions et concepts du programme.

[...] Il est à noter que les genres ont orienté le choix des phénomènes linguistiques et textuels qui font l'objet d'un apprentissage systématique. (Ministère de l'Éducation, du Loisir et du Sport, 2011a, p. 4)

Pour chacun de ces éléments, certains apprentissages spécifiques sont attendus selon les deux cycles du secondaire. La différence tient, selon les termes des textes officiels, à un degré de complexité croissant: "D'une année à l'autre, les élèves sont amenés à explorer des textes écrits et oraux de plus en plus complexes dans des contextes diversifiés et stimulants » (ibid.). La complexité concerne principalement, dirons-nous, le « degré de transparence » des informations contenues dans les textes (par exemple, des informations explicites sur les personnages et les lieux aux informations implicites) ainsi que les éléments constitutifs de la «mécanique narrative ». Ainsi passe-t-on, à titre illustratif, de la capacité à distinguer l'auteur du narrateur ( $3^{\mathrm{e}}$ secondaire) à celle de repérer le type de narrateur unique (omniscient ou participant à l'histoire, $4^{\mathrm{e}}$ secondaire) pour arriver à la capacité d'identifier des narrateurs multiples ( $5^{\mathrm{e}}$ secondaire). Mais la complexité concerne aussi d'autres dimensions du texte comme ce qui touche à son contexte de production et de diffusion. Au premier cycle du secondaire, les élèves doivent être capables de tenir compte, pour orienter leur lecture d'un texte, des éléments suivants : «La date de production ou de publication, l'édition, la collection, le lieu». Au second cycle, il est attendu qu'ils sachent distinguer «le contexte historique ou socioculturel de production ([...] l'époque durant laquelle se déroule le récit)» (ibid., p. 29).

De manière plus générale, pour ce qui concerne la compétence en lecture, la progression attendue du primaire au secondaire est présentée de la sorte :

Au primaire, l'élève a lu et écrit des textes qui racontent des histoires. Au cours de ses lectures, il s'est familiarisé avec certains genres de textes dans la littérature pour la jeunesse d'ici et d'ailleurs. Il a développé des stratégies de lecture: la recherche des éléments constitutifs d'une histoire et la création de liens entre les actions et les motivations des personnages. Il a souvent eu l'occasion de réagir aux textes en partageant ses impressions et en établissant des liens avec ses expériences personnelles ou d'autres textes. [...]

$\mathrm{Au} 1^{\mathrm{er}}$ cycle du secondaire, l'élève est amené à se représenter la narration comme l'action de raconter comment, au cours d'une suite d'évènements, un ou des personnages réussissent ou non une quête d'équilibre. Il apprend à recourir de manière autonome au schéma narratif pour comprendre l'histoire ; il s'intéresse au narrateur et à la manière dont l'histoire est racontée. Soutenu par des échanges avec l'enseignante ou l'enseignant et ses pairs, il découvre que des interprétations multiples, créées autour de réseaux de sens, peuvent être fondées. [...]

$\mathrm{Au} 2^{\mathrm{e}}$ cycle, attentif à ce qu'il apprend sur la langue, le texte et la culture, l'élève peut entrer dans des textes plus exigeants et développer davantage qu'au $1^{\text {er }}$ cycle sa capacité à les apprécier. En fréquentant des œuvres de la littérature québécoise, de la francophonie et du patrimoine mondial, l'élève élargit sa vision du monde ; il 
comprend mieux l'actualité ou l'universalité des thèmes traités et découvre que

l'appartenance à une communauté et à une culture est marquée. (ibid., p. 27)

19 Au primaire, pour lire et apprécier des œuvres littéraires, diverses connaissances et stratégies sont présentées dans la Progression des apprentissages: explorer des œuvres variées, recourir aux œuvres littéraires à diverses fins, etc. Cela dit, les activités didactiques de lecture et d'appréciation à mettre en place, en classe du primaire, demeurent à la discrétion des enseignant·e·s.

$\mathrm{Au}$ secondaire, le programme du $2^{\mathrm{e}}$ cycle insiste sur l'importance de proposer aux élèves des tâches qui les amènent à interagir entre lecteurs : « Pour soutenir les élèves dans leur travail d'appréciation de même que dans les défis qui les conduisent à entrer avec plus de profondeur dans les textes et à lire des textes plus exigeants, l'enseignant doit leur proposer des tâches qui les placent fréquemment en situation d'interagir avec leurs pairs » (Ministère de l'Éducation, du Loisir et du Sport, 2009b, p. 28). Voilà une prescription qui valorise la dimension intersubjective de la lecture et ouvre la porte à la mise en place de communautés interprétatives (Sauvaire, 2017).

21 Le programme du collégial, jusqu'en 2012, ne donnait aucune information sur les activités d'apprentissage à privilégier, mais la liste de "critères de performance » stipule que les apprenants doivent, au terme du premier cours, par exemple, être à même de "repérer et classer des thèmes et des procédés stylistiques ", d'établir des «liens pertinents entre le propos du texte, les manifestations thématiques et les manifestations stylistiques » et de faire la différence entre « des idées principales et des idées secondaires [dans un] plan de rédaction " en vue de "[r]édiger et réviser une analyse littéraire ou un commentaire composé ou une explication de texte » (Ministère de l'Éducation du Québec, 1998, p. 15). Ces éléments sont susceptibles de guider le travail d'analyse des textes à réaliser. À cette étape finale de la formation scolaire du lecteur de textes littéraires, les instructions officielles comprennent des orientations qui laissent à priori peu de place au sujet lecteur, privilégiant une approche objectivante et pouvant se traduire par des pratiques qui, selon S. Ouellet (2012, p. 11), « nuisent à l'appropriation du phénomène littéraire » parce qu'elles maintiennent les élèves « dans un rapport extérieur avec l'œuvre littéraire par des exercices de lecture analytique et la rédaction de dissertations ».

22 Le concept d'approche analytique n'est repris de manière explicite dans aucun des programmes québécois. Le terme "analyse» est utilisé principalement dans ces documents en rapport avec le travail systématique à réaliser sur la langue (analyse d'une phrase, de la morphologie d'un mot) mais aussi pour ce qui a trait à l'analyse des situations de communication dans lesquelles sont produits les discours étudiés. Sans préciser ce que cela recouvre exactement, le programme du secondaire retient parmi les paramètres à considérer pour la progression des apprentissages du début à la fin du secondaire, comme indiqué précédemment, la «complexité » du texte qui va de pair avec des niveaux différents de "profondeur d'analyse »: "d'un texte de complexité moyenne à un texte présentant ou exigeant une certaine profondeur d'analyse, jusqu'à un texte consistant sollicitant de nombreuses ressources des élèves (grande capacité de lecture, connaissances générales étendues, repères culturels de divers ordres » (Ministère de l'Éducation, du Loisir et du Sport, 2009b).

23 Par rapport à ce curriculum prescrit, que sait-on des choix effectués par les enseignant·es et des pratiques qu'ils privilégient dans leurs classes en fonction des 
publics avec lesquels ils travaillent au quotidien? C'est ce que nous tentons d'éclairer dans la partie suivante.

\section{Des données empiriques multiples pour une tentative de portrait d'ensemble} atives aux choix des œuvres complètes et aux activités associées à la lecture de ces œuvres a porté, à la fois sur le dernier cycle du primaire et sur les deux cycles du secondaire ${ }^{8}$. Les résultats obtenus à travers une enquête par questionnaire, à laquelle ont répondu 291 enseignant·e's du dernier cycle du primaire et 392 enseignant·e's des deux cycles du secondaire, ont été complétés par des entrevues réalisées auprès de 45 enseignant·es. L'examen de la situation s'est poursuivi entre 2009 et 2012 pour ce qui concerne l'ordre collégial'. Cette recherche a porté sur l'analyse de 413 plans de cours ainsi que sur les réponses obtenues à une enquête par questionnaire $(n=95)$ et lors d'entrevues avec des enseignant·es $(n=20)$. Des observations ont aussi été effectuées dans des classes. J. Babin (2016) a exploité ces données dans une étude multicas afin de décrire des pratiques d'enseignement sous la loupe de ce qui s'enseigne (angles d'entrée dans l'œuvre, savoirs privilégiés) et la façon dont cela est fait (rôles tenus par les enseignant·e·s).

\section{Les formats et les corpus}

Du primaire au collégial, on remarque une présence importante et continue des œuvres complètes aux côtés des extraits. Selon les données de l'enquête d'o. Dezutter, au dernier cycle du primaire, environ un enseignant sur cinq (17 \%) affiche des exigences très élevées en termes quantitatifs, avec un programme de lecture qui comporte dix œuvres (albums et petits romans) ou plus par année. Au secondaire, la moyenne du nombre d'œuvres complètes à lire chaque année est entre 4 et $5(4,72)$. Au collégial, le 
programme de lecture comporte selon les données recueillies en moyenne 3 œuvres par cours, le programme imposant 8 œuvres pour les 3 cours.

Selon l'enquête de M. Lépine, au primaire, la proportion d'œuvres complètes est nettement supérieure aux extraits. Par exemple, deux tiers (66,2\%) des enseignant·es sondée's disent utiliser des œuvres littéraires intégrales en papier au moins une fois par semaine, tandis qu'un sur dix (13,9\%) déclare se servir d'extraits d'œuvres littéraires en papier de façon hebdomadaire. L'utilisation des œuvres littéraires intégrales en format papier est plus fréquente chez les personnes répondantes enseignant au premier cycle $(81,1 \%)$ que chez celles enseignant au deuxième cycle $(61,2 \%)$ et au troisième cycle $(52,3 \%)$.

Plus on avance dans la scolarité, plus la liberté laissée aux élèves pour le choix des titres diminue. Dans l'enquête d'o. Dezutter, une enseignante sur deux au dernier cycle du primaire laisse une liberté totale de choix ou n'impose que quelques critères généraux de sélection (Dezutter et al., 2012). Cela recoupe les données recueillies par M. Lépine (2017): la presque totalité des enseignante's du primaire interrogée's $(97,7 \%)$ disent offrir, au moins toutes les semaines, une période de lecture personnelle où les élèves sont libres de lire les livres de leur choix, et ce, très souvent pour s'occuper une fois un travail terminé $(93,4 \%)$. Près de quatre enseignants sur cinq $(77,0 \%)$ déclarent proposer tous les jours des moments de lecture libre. Lorsque nous analysons les résultats par cycle, nous constatons que la lecture personnelle libre est une pratique hebdomadaire quasi systématique pour les enseignants des trois cycles ( $98,8 \%$ au premier cycle, $97,8 \%$ au deuxième et $96,4 \%$ au troisième).

30 Au secondaire, même si le programme du deuxième cycle invite à ce que le choix des œuvres soit partagé entre les élèves et les enseignants (Ministère de l'Éducation, du Loisir et du Sport, 2009b), ce sont plus des trois quarts des répondant·es qui ont déclaré imposer la totalité ou la majorité des titres du programme de lecture annuel. Cette proportion augmente encore au collégial, où la presque majorité des enseignant·e·s $(92,9 \%)$ imposent tous les titres à lire. À cet ordre d'enseignement, cela s'explique probablement par les orientations du programme, qui présentent l'enjeu majeur des cours de « Français, langue et littérature » comme étant de contribuer à faire connaitre "les œuvres et les textes littéraires qui ont marqué l'histoire de la littérature " (Ministère de l'Éducation, du Loisir et du Sport, 2009b, p. 6).

31 En termes de genres, plus les élèves avancent dans leur parcours scolaire, plus ils sont mis en contact avec une diversité de genres. Au primaire, selon les répondantees à l'enquête de M. Lépine, l'album ( $81,3 \%)$, le roman (61,2 \%) et le conte $(55,3 \%)$ sont les trois formes/genres littéraires que les enseignantees déclarent le plus souvent utiliser au primaire, que ce soit en tant qu'œuvres intégrales ou extraits. Les enseignantees du premier cycle $(97,1 \%$,) disent davantage se servir des albums que leurs collègues des deuxième $(78,5 \%)$ et troisième cycles $(64,3 \%)$. Le roman est utilisé davantage au troisième cycle $(91,6 \%)$ et au deuxième cycle $(79,1 \%)$ qu'au premier cycle $(23,8 \%)$. Le conte est exploité plus souvent au premier cycle $(67,9 \%$ ) qu'au deuxième $(58,3 \%)$ et qu'au troisième cycle $(36,9 \%)$. Par ailleurs, la chanson (19,9\%), la légende $(18,3 \%)$, le poème $(14,7 \%)$, la fable $(12,7 \%)$ et la bande dessinée $(11,4 \%)$ sont moins utilisés par les enseignant·e's. La nouvelle littéraire (15,0 \%), la comptine $(12,5 \%)$ et la pièce de théâtre (6,5\%) sont aussi moins exploitées, environ le tiers des enseignant·es ayant affirmé ne jamais les utiliser. 
32 Au secondaire, même si le programme d'études et la Progression des apprentissages demandent de travailler sur des textes poétiques et dramatiques, ceux-ci ne sont abordés que sous la forme d'extraits dans la majorité des cas selon O. Dezutter et al. (2007). Pour le format œuvre complète, c'est le roman qui est omniprésent. Ceci peut être mis en relation avec les éléments d'analyse narratologique repérés dans le programme.

33 La situation change au collégial, où les étudiant·e's expérimentent une plus grande diversité de genres. Au vu de l'analyse des plans de cours réalisée par O. Dezutter et al. (2012), deux tiers des étudiant·es du collégial ont à lire une pièce de théâtre en intégralité $(68,7 \%$ des plans de cours), et un recueil de poésie apparait dans le programme de lecture d'une étudiant·e sur cinq (19,5\%). La lecture de l'essai reste marginale $(5,9 \%)$.

34 Enfin, pour ce qui concerne les auteur'e's et les titres phares, tant au primaire qu'au secondaire, on observe un fort éclatement du corpus. Par exemple, seulement

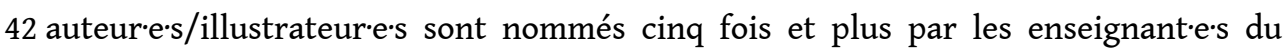
primaire, et des centaines le sont moins de cinq fois. L'auteure la plus citée, D. Demers, revient sur moins d'un questionnaire sur cinq (16,4\%). Il y aurait donc une grande diversité d'auteur·e's particulièrement appréciés des enseignant·e·s du primaire, avec une prédominance des auteur·e's québécois·e·s.

$35 \mathrm{Au}$ secondaire, les deux titres ayant recueilli le plus de mentions dans l'enquête d'O. Dezutter - La Route de Chlifa, œuvre contemporaine d'une auteure québécoise phare de la littérature pour adolescents, Michèle Marineau, et le grand classique international Le Petit Prince - ne sont imposés chacun que par 1,1\% des enseignants et sont lus plus souvent au premier cycle. Les enseignants du secondaire semblent donc profiter pleinement de la liberté laissée par le ministère en matière de corpus à faire découvrir aux élèves.

\section{Les activités}

36 À l'école primaire, plusieurs activités ont pour principal objectif affiché d'installer la pratique de la lecture des œuvres complètes, qui se présentent sous des formats divers (album, recueil de contes ou de poésies, mini-romans), et d'augmenter l'intensité de cette pratique. En ce sens, selon l'enquête de M. Lépine, des activités portées par l'enseignant.e sont mises en œuvre chaque semaine. Près des deux tiers des enseignant.e.s $(61,1 \%)$ disent faire la lecture à voix haute à leurs élèves chaque semaine ; de ce nombre, un sur cinq $(19,6 \%)$ le fait même tous les jours. De façon hebdomadaire, près d'un·e enseignant·e sur cinq (17,3\%) a indiqué faire découvrir à ses élèves des livres audio.

D'autres activités portées davantage par les élèves sont aussi très fréquentes. La presque totalité des enseignant·e·s interrogé·e·s (97,7 \%) disent offrir, au moins toutes les semaines, une période de lecture personnelle où les élèves sont libres de lire les livres de leur choix. Près de quatre enseignant-e's sur cinq $(77,0 \%)$ déclarent proposer tous les jours des moments de lecture libre. Durant ces périodes de lecture personnelle, les élèves peuvent lire des œuvres littéraires, mais aussi d'autres types de livres et de textes. Quant à la lecture d'une même œuvre littéraire imposée à tous les élèves d'un groupe, environ le quart des enseignant·es $(27,8 \%)$ déclarent en exiger une chaque 
semaine. Une personne enseignante sur deux $(51,3 \%)$ a indiqué imposer la lecture d'œuvres littéraires en devoir de façon hebdomadaire.

En reprenant la distinction entre activités métatextuelles et hypertextuelles proposée par C. Simard et al. (2010) ainsi que par B. Schneuwly et al. (2017), il apparait que les enseignante's rapportent faire davantage d'activités métatextuelles que d'activités hypertextuelles. De façon globale, selon les données recueillies par M. Lépine, en ce qui concerne les exploitations écrites, moins de la moitié des enseignante-s décrivent des activités métatextuelles $(43,1 \%)$; le tiers, des activités hypertextuelles $(31,0 \%)$; et le cinquième, des activités offrant une combinaison des deux (21,6\%). Les activités d'écriture mises en place par les enseignantees et réalisées par les élèves se font généralement en situation individuelle, mais plusieurs enseignant·e's déclarent aussi organiser ces activités en petites équipes ou encore sous forme d'écriture collective impliquant toute la classe, et ce, de façon plus marquée pour les activités hypertextuelles.

Toujours concernant le primaire, les activités emblématiques à l'oral, contrairement aux activités d'écriture, apparaissent plus spontanées et informelles: "Ce sont des activités informelles la plupart du temps. Chaque jour, je leur lis une histoire. Avant, pendant et après ma lecture, je questionne mes élèves sur ce qu'ils pensent de l'histoire, sur ce qu'ils croient qu'il va arriver, etc.» (enseignant 521-c1 dans Lépine, 2017). Le travail autour des textes est ici centré sur la compréhension de l'intrigue et sur les réactions des élèves.

Peu importe le cycle analysé, les discussions autour d'une lecture, les présentations orales de coups de cœur littéraires et les cercles de lecture sont très souvent cités par les enseignant-e's comme activités représentatives de leur enseignement qui s'appuie donc fortement sur une approche intersubjective. De façon globale, les résultats obtenus en ce qui concerne les exploitations orales sont nettement en faveur des activités métatextuelles: la quasi-totalité des enseignant-e's décrivent des activités métatextuelles $(92,7 \%)$, et moins d'un sur vingt des activités hypertextuelles $(2,3 \%)$ ou une combinaison des deux (1,5\%).

41 Tant au primaire qu'au secondaire, l'activité métatextuelle par excellence est la passation du questionnaire visant à vérifier la compréhension de l'œuvre lue. Nous avons déjà souligné que le recours à ce type de questionnaire s'inscrit très probablement en partie dans un souci de préparer les élèves aux épreuves ministérielles portant sur l'évaluation des compétences en lecture. Un exemple d'activité hypertextuelle à l'écrit est la tenue d'un carnet ou journal de lecture, une pratique que M. Brunel (2013) range parmi celles susceptibles de solliciter une posture de sujet lecteur, mais qui est davantage déclarée par les enseignant-ess du primaire que par celles et ceux du secondaire (Dezutter et al., 2012). La plupart des activités métatextuelles ont une fonction d'évaluation. C'est que dans le contexte scolaire, une fois la pratique de lecture installée, la lecture d'une œuvre devient indissociable d'une activité (Bergeron et al., 2005) et d'une dimension évaluative.

\section{Les modes d'évaluation}

42 Les enseignant·es du primaire interrogés par M. Lépine (2017) disent évaluer souvent chez leurs élèves, en priorité, la compréhension (90,0\%), ensuite la réaction $(72,8 \%)$, l'interprétation $(65,5 \%)$, les connaissances/stratégies $(65,3 \%)$ et le jugement critique 
$(55,0 \%)$. De façon globale, pour les outils d'évaluation à l'écrit, près de la moitié des enseignante-s disent utiliser au moins tous les mois les cahiers, journaux ou carnets $(46,6 \%)$, les examens provenant de banques diverses $(45,3 \%)$ et les questionnaires maison (43,4\%). De façon mensuelle, très peu d'enseignante-s se servent des épreuves ministérielles $(2,2 \%)$ qui sont davantage réservées aux évaluations en fin d'année. Cela étant, les cahiers, journaux ou carnets des élèves sont utilisés par près d'une enseignante sur cinq $(19,8 \%)$ chaque semaine. À l'oral, le tiers des enseignante-s déclare organiser tous les mois des groupes de discussion $(36,0 \%)$ et des entretiens individuels $(33,4 \%)$. Une enseignante sur cinq $(20,1 \%)$ affirme d'ailleurs amener ses élèves à faire des présentations orales chaque mois.

O. Dezutter et C. Morissette (2010) ont interrogé deux groupes d'élèves de première année du secondaire à propos de leurs perceptions relatives aux activités imposées en lien avec la lecture des œuvres complètes dans le contexte scolaire. Les enseignantes de ces groupes avaient demandé aux élèves de réaliser durant l'année scolaire la lecture de quatre œuvres et de réaliser les travaux suivants, tous objets d'évaluation: un exposé oral en groupe comprenant des informations sur l'auteur et un résumé du premier livre lu, imposé par l'enseignante; un résumé écrit de la deuxième œuvre choisie individuellement par chaque élève; une critique de la troisième œuvre lue, choisie aussi individuellement ; et enfin un résumé et une critique écrits de la quatrième œuvre elle aussi résultant d'un choix individuel.

Les trois quarts des élèves $(77,2 \%)$ ont estimé que ces activités, dont la moitié sont centrées sur la composante " apprécier » et le jugement critique, étaient utiles ou très utiles pour leurs apprentissages. Une différence nette apparait toutefois entre les élèves qui préfèrent les activités écrites et ceux qui apprécient davantage les activités orales.

Compte tenu de l'objectif principal de chacun des cours au collégial - écrire une analyse ou une dissertation -, tel qu'indiqué lors de la présentation des différents programmes, les évaluations rapportées par les enseignante's de cet ordre d'enseignement dans l'enquête d'O. Dezutter et al. sont très souvent liées à une activité de rédaction: dans $65 \%$ des plans de cours analysés et dans $100 \%$ des questionnaires autodéclarés, les enseignante-s prévoient d'évaluer la lecture en exigeant des apprenante-s un texte sur les procédés littéraires récurrents dans l'œuvre (ou un extrait de celle-ci) et sur les effets de ces procédés (Babin et al., 2012). On est dans ce cas dans une approche objectivante et une forme de lecture savante. La notion de "contrôle » de lecture est également présente lorsqu'il est question d'évaluation. Dans près de la moitié des plans de cours $(48 \%)$ et selon plus de trois quarts des repondante's à l'enquête, un questionnaire visant à vérifier la lecture effective de l'œuvre ou la compréhension de celle-ci est planifié (ibid.).

\section{Conclusion}

L'examen du curriculum prescrit et les informations disponibles sur le curriculum enseigné présentées dans cet article montrent que les pratiques d'enseignement de la lecture des textes littéraires dans les classes québécoises présentent certes une grande variété mais aussi certains traits communs et des caractéristiques propres à chaque ordre d'enseignement. En termes de contexte de lecture, alors que les premières expériences consistent la plupart du temps en des lectures partagées collectivement 
dans le temps scolaire, se met aussi très vite en place la volonté de faire de la lecture des œuvres une activité externe au temps scolaire et autonome. Cette exigence s'affirme au fur et à mesure de la scolarité et les choix de lecture sont de plus en plus imposés. En termes de finalités, pour reprendre le cadre de S. Richard (2006), on passe progressivement d'une centration sur les finalités d'ordres psychoaffectif et cognitivolangagier à des finalités d'ordre esthético-culturel. Cela va de pair avec une variation progressive des contenus travaillés qui passent, des savoirs sur la langue et sur les processus de lecture, à des savoirs sur le monde et la littérature (Babin, 2016). Le mode de lecture privilégié, qui relève d'abord d'une lecture subjective, évolue vers une lecture savante (Simard et al., 2010). Enfin, de manière générale, on peut considérer que le travail engagé sur les textes littéraires conduit les élèves à intégrer deux types d'habitus. Le premier habitus concerne la valorisation de la pratique de la lecture des œuvres comme une pratique sociale partagée, une pratique que l'école contribue à installer, même si le milieu familial et les pairs jouent en ce sens aussi un rôle essentiel. Le second habitus est d'ordre scolaire, il porte sur une forme de disciplination des élèves (Ronveaux \& Schneuwly, 2019) qui les amène à intégrer progressivement le fait que la lecture des textes littéraires à l'école s'inscrit dans des objectifs spécifiques et qu'elle s'accompagne d'une série d'activités types qui font pour la plupart, l'objet d'une évaluation.

\section{BIBLIOGRAPHIE}

BABIN, J. (2016). La lecture d'œuvres littéraires complètes au collégial : des cas de pratiques d'enseignement dans le cours d'Écriture et Littérature. Thèse de doctorat en éducation : Université de Sherbrooke. En ligne : https://savoirs.usherbrooke.ca/bitstream/handle/11143/9782/

Babin_Julie_PhD_2016.pdf?sequence=1\&isAllowed=y.

BABIN, J. et al. (2012). « La place des œuvres littéraires complètes dans les cours de français des collèges du Québec. Quelle progression d'un cours à l'autre ? "In : Dumortier, J.-L., Van Beveren, J. \& Vrydaghs, D (dirs). Curriculum et progression en français. Actes du $11^{e}$ colloque de l'AiRDF (Liège, 26-28 aout 2010). Namur : Presses universitaires de Namur.

BRUNEL, M. (2013). « Le sujet lecteur dans la classe : éléments pour un état des lieux des pratiques dans le secondaire ». Recherches \& Travaux 83, p. 117-130. En ligne : https://

journals.openedition.org/recherchestravaux/655.

CANVAT, K. (2005). « De l'enseignement à l'apprentissage de la littérature ou des savoirs aux compétences ». In : Brillant-Annequin, A. \& Massol, J.-F. (dirs). Le Pari de la littérature. Quelles littératures de l'école au lycée?. Grenoble : Scéren-CRDP, p. 29-41.

CHARTRAND, S.-G., ÉMERY-BRUNEAU, J., \& SÉNÉCHAL, K. (2015) [2013]. Caractéristiques de 50 genres pour développer les compétences langagières en français. Québec : Didactica-C.É.F.

DEZUTTER, O. et al. (2007). « Les pratiques déclarées des enseignants québécois dans la sélection et l'exploitation des œuvres complètes inscrites au programme de lecture des élèves ». In : 
Falardeau, É. et al. (dirs). La Didactique du français. Les voies actuelles de la recherche. Québec: Presses de l'université Laval, p. 83-100.

DEZUTTER, O., et al. (2012). «La lecture des œuvres complètes en contexte scolaire au Québec. État des lieux ». Revue internationale d'éducation de Sèvres 61, p. 111-120. En ligne : https://

journals.openedition.org/ries/2690.

DEZUTTER, O. \& MORISSETTE, C. (2010). « Que pensent des élèves québécois des œuvres à lire en contexte scolaire et des activités imposées autour de ces lectures?». In : Louichon, B. \& Rouxela, A. (dirs). Du corpus scolaire à la bibliothèque intérieure. Rennes : Les presses de l'université de Rennes, p. 201-209.

FLOREY, S. et al. (dirs) (2015). Enseigner la littérature au début du XXI e siècle. Enjeux, pratiques, formation. Bruxelles : P. Lang.

GENETTE, G. (1972). Figures III. Paris : Éd. du Seuil.-

GREIMAS, A. J. (1966). « Éléments pour une théorie de l'interprétation du récit mythique ». Communications 8, p. 28-59. En ligne : https://www.persee.fr/doc/

comm_0588-8018_1966_num_8_1_1114.

LÉPINE, M. (2017). L'Enseignement de la lecture/appréciation des œuvres littéraires à l'école primaire: enquête sur les pratiques déclarées et les conceptions d'enseignants québécois. Thèse de doctorat en éducation : Université de Montréal. En ligne : https://papyrus.bib.umontreal.ca/xmlui/ bitstream/handle/1866/20052/Lepine_Martin_2017_these.pdf?sequence=3\&isAllowed=y?

Ministère de l'Éducation, de l'Enseignement supérieur et de la Recherche (2015). Épreuve uniforme de français, langue d'enseignement et littérature. Collégial. Québec : Gouvernement du Québec. En ligne : http://www.education.gouv.qc.ca/colleges/etudiants-au-collegial/epreuves-de-langue/ epreuve-uniforme-de-francais/.

Ministère de l'Éducation, du Loisir et du Sport (2001). Programme de formation de l'école québécoise (éducation préscolaire et enseignement primaire). Québec : Gouvernement du Québec. En ligne : https://numerique.banq.qc.ca:443/patrimoine/details/52327/41070?docpos=4.

Ministère de l'Éducation, du Loisir et du Sport (2006). Programme de formation de l'école québécoise ( $1^{\text {er }}$ cycle du secondaire). Québec : Gouvernement du Québec. En ligne : http:// www.education.gouv.qc.ca/fileadmin/site_web/documents/PFEQ/prfrmsec1ercyclev2.pdf.

Ministère de l'Éducation, du Loisir et du Sport (2009a). Formation générale commune, propre et complémentaire aux programmes d'études conduisant au diplôme d'études collégiales. Québec : Gouvernement du Québec. En ligne : https://numerique.banq.qc.ca/patrimoine/details/ 52327/1944836?docref=2v2kOo4D6fK3MX2kD_uQ1g.

Ministère de l'Éducation, du Loisir et du Sport (2009b). Programme de formation de l'école québécoise ( $2^{e}$ cycle du secondaire). Français, langue d'enseignement. Québec: Gouvernement du Québec. En ligne : http://www.education.gouv.qc.ca/fileadmin/site_web/documents/dpse/ formation_jeunes/PFEQ_FrancaisLangueEnseignement.pdf.

Ministère de l'Éducation, du Loisir et du Sport (2009c). Progression des apprentissages au primaire. Français, langue d'enseignement. Québec : Gouvernement du Québec. En ligne : http:// www.education.gouv.qc.ca/fileadmin/site_web/documents/education/jeunes/pfeq/ PDA_PFEQ_francais-langue-enseignement-primaire_2011.pdf.

Ministère de l'Éducation, du Loisir et du Sport (2011a). Progression des apprentissages au secondaire. Français, langue d'enseignement. Québec : Gouvernement du Québec. En ligne : http:// 
www.education.gouv.qc.ca/fileadmin/site_web/documents/education/jeunes/pfeq/ PDA_PFEQ_francais-langue-enseignement-secondaire_2011.pdf.

Ministère de l'Éducation, du Loisir et du Sport (2011b). Cadre d'évaluation des apprentissages. Français, langue d'enseignement. Primaire - $1^{e r}, 2^{e}$ et $3^{e}$ cycle. Québec : Gouvernement du Québec.

Ministère de l'Éducation du Québec (1980). Programme d'études. Français, langue maternelle. Québec : Gouvernement du Québec.

Ministère de l'Éducation du Québec (1998). Programme de formation générale commune. Langue d'enseignement et littérature. Québec : Gouvernement du Québec.

OUELLET, S. (2012). Le Sujet lecteur et scripteur : développement d'un dispositif didactique en classe de littérature. Thèse en éducation : Université du Québec à Montréal/Université de Toulouse II-Le Mirail.

POPHAM, J. W. (2001). « Teaching to the Test? » Educational Leadership 58 (6), p. 16-20. En ligne : http://www.ascd.org/publications/educational-leadership/mar01/vol58/num06/Teaching-tothe-Test $₫$.aspx.

RICHARD, S. (2006). « Analyse des discours didactiques concernant les finalités de l'enseignement de la littérature au secondaire de 1970 à $2004 »$. In : Falardeau, É. et al. (dirs). Acte du $9^{e}$ colloque de l'Association internationale pour le développement de la recherche en didactique du français, Québec 26-28 août 2004. En ligne : http://www.colloqueairdf.fse.ulaval.ca/fichier/Communications/SuzanneRichard.pdf.

RONVEAUX, C., \& BABIN, J. (2020). « CEuvre intégrale ». In : N. Brillant Rannou, F. et al. (dirs.). Un dictionnaire de didactique de la littérature. Paris : H. Champion, p. 309-311.

RONVEAUX, C. \& SCHNEUWLY, B. (2019). « Discipliner par la littérature. Questions et options méthodologiques pour une approche descriptive et explicative quasi expérimentale en milieu écologique ». In : Denizot, N., Dufays, J.-L. \& Louichon, B. (dirs). Approches didactiques de la littérature. Namur : Presses universitaires de Namur, 2019. p. 139-156.

SAUVAIRE, M. (2017). « Entre errance et erreur : la diversité des lectures subjectives à l'épreuve de la communauté interprétative ». In : L'Enseignement et l'apprentissage de la lecture aux différents niveaux de la scolarité. Namur : Presses universitaire de Namur, p. 297-329. En ligne : https:// books.openedition.org/pun/5143?lang=fr.

SCHNEUWLY, B. et al. (2017). « Writing and teaching literature the role of hypertextual and metatextual writing activities at three school levels ». L1 Educational studies in languages and literature 16-17. En ligne : https://archive-ouverte.unige.ch/unige:93165.

SIMARD, C., et al. (2010). Didactique du français langue première. Bruxelles/Montréal : De Boeck/ Éditions du Renouveau pédagogique.

\section{NOTES}

1. «Est œuvre intégrale toute production textuelle singulière, produite par un auteur, délimitée par sa matérialité d'objet-livre, par son unité thématique, par l'organisation de son contenu [...]» (Ronveaux \& Babin, 2020, p. 309).

2. Au Québec, le diplôme d'études collégiales est un diplôme postsecondaire. À ce niveau de formation, deux types de filières existent : l'une est directement qualifiante 
(formation technique) et l'autre est une préparation aux études universitaires (filière générale).

3. Les programmes de français du Québec établissent une différence entre les textes courants et les textes littéraires. Les textes courants ont une visée principalement utilitaire et peuvent être de type descriptif, argumentatif, explicatif ou justificatif.

4. Au primaire, les cycles sont de deux années. Au secondaire, le $1^{\text {er }}$ cycle compte deux années et le $2^{\mathrm{e}}$ cycle, trois.

5. Le phénomène est connu dans le monde anglophone comme le "teach to the test ", où les responsables de cours visent avant tout à faire réaliser aux apprenant·e's des tâches dans des situations calquées sur celles qui seront rencontrées lors de l'évaluation, notamment en formulant des consignes très similaires (Popham, 2001).

6. La discipline "français", au secondaire, avait déjà adopté cette approche dès les programmes de 1995.

7. Depuis 2011, dans le bulletin unique, ces deux compétences pour le primaire ont été reformulées en une seule : " Lire et apprécier des œuvres littéraires. »

8. Plusieurs publications ont découlé de ce projet de recherche. Voir notamment Dezutter et al. (2007).

9. Voir entre autres Babin et al. (2012).

\section{RÉSUMÉS}

Dans cette contribution, nous partageons, tout d'abord, notre vision de la spécificité du rapport aux textes littéraires en contexte scolaire. Après cela, nous proposons un regard analytique sur la manière dont les programmes de français québécois des ordres d'enseignement primaire, secondaire et collégial encadrent la lecture des textes littéraires et le travail effectué autour de ces textes, dont le mode d'approche à privilégier, en essayant de dégager certains indices de progression à travers les différents ordres et cycles d'enseignement. En écho aux éléments du curriculum prescrit, nous présentons les résultats saillants d'un ensemble de données empiriques recueillies dans le cadre de plusieurs recherches étalées sur les vingts dernières années et portant principalement sur les pratiques déclarées des enseignant·e·s du primaire, du secondaire et du collégial.

In this contribution, we first share our vision of what makes teaching of literary text reading distinctive. We also offer an analytical look at the way in which Quebec French programs at the primary, secondary and college levels frame the act of reading literary texts and the school work around these texts, looking for certain signs of progress. Echoing the elements of the prescribed curriculum, we finally present some salient results of several researches spread over the last fifteen years, from which we gathered a set of empirical data on the practices of K-12 teachers. 
INDEX

Mots-clés : Euvre complète, littérature, progression, didactique de la lecture, Québec

Keywords : Literary work, literature, progress, reading didactics, Quebec

\section{AUTEURS}

\section{OLIVIER DEZUTTER}

Université de Sherbrooke, Collectif CLÉ, QC J1K 2R1 Sherbrooke, Canada

JULIE BABIN

Laboratoire CRIFPE, Université Laval, QC G1V 0A6 Québec, Canada

\section{MARTIN LÉPINE}

Université de Sherbrooke, Collectif CLÉ, QC J1K 2R1 Sherbrooke, Canada 